\title{
Fisiologia digestiva de suínos alimentados com rações contendo diferentes fontes de fósforo
}

\author{
José Aparecido Moreira², Dorinha Miriam Silber Schmidt Vitti ${ }^{3}$, Alexandre de Oliveira \\ Teixeira $^{4}$, João Batista Lopes ${ }^{2}$
}

\footnotetext{
${ }_{1}^{1}$ Projeto financiado pela FAPESP-06/57574-5 e 04/14532-5.

2 Pós-Doutorando do Laboratório de Nutrição Animal-CENA/USP.

${ }^{3}$ Laboratório de Nutrição Animal-CENA/USP, Piracicaba, SP.

${ }^{4}$ Departamento Técnico-BUNGE Fertilizantes $S / A$.

${ }^{5}$ Departamento de Zootecnia-UFPI, Teresina, $\mathrm{PI}$.
}

RESUMO - Objetivou-se avaliar a fonte de fósforo de melhor qualidade para uso na alimentação de suínos e que provoque menor impacto ambiental pela excreção de fósforo no ambiente. Foram utilizados 21 suínos machos castrados com peso inicial de $31,94 \pm 2,88 \mathrm{~kg}$ mantidos com dietas à base de milho, farelo de soja e óleo vegetal (controle) suplementadas com fosfato bicálcico (FBC), fosfato monobicálcico (MBC), superfosfato triplo (ST), superfosfato simples (SS), ácido fosfórico (AcF) ou fosfato de rocha catalão. O delineamento experimental foi o de blocos casualizados, com sete fontes de fósforo e três repetições e um animal por unidade experimental. O modelo usado foi determinístico e compartimental, de modo que o trato gastrintestinal $\left(\mathrm{C}_{1}\right)$, o plasma $\left(\mathrm{C}_{2}\right)$, os ossos $\left(\mathrm{C}_{3}\right)$ e os tecidos moles $($ coração, fígado, rins e músculos) estudados em conjunto $\left(\mathrm{C}_{4}\right)$ representaram os compartimentos. Foram utilizados dados de metabolismo e cinética do fósforo em tecidos, obtidos pela técnica de diluição isotópica. O superfosfato simples destacou-se entre as fontes estudadas como a fonte com menor capacidade de poluir o meio ambiente, melhor biodisponibilidade e metabolismo mais eficiente em suínos em crescimento.

Palavras-chave: diluição isotópica, fósforo endógeno, metabolismo, modelo biomatemático, radiofósforo

\section{Digestive physiology of swine fed diets with different phosphorus sources}

ABSTRACT - The source of phosphorus of better quality for use in the pig feeding and that cause less environment impact by the excretion of phosphorus in the enviroment was evaluated. Twenty-one male pigs weighting $34.94 \pm 2.88$ were fed corn-soybean meal based diets supplemented with different $\mathrm{P}$ sources: control without $\mathrm{P}$ supplementation $\mathrm{P}$ (Cont), dicalcium (FBC), monodicalcium (MBC), triple superphosphate (ST), simple superphosphate (SS), Catalão rock (Rocha) and phosphoric acid (AcF). The experiment was designed to evaluate the $\mathrm{P}$ availability and $\mathrm{P}$ excretion to environment. The experiment was arranged in randomized blocks, with seven treatments and three replicates and one pig/box. The model was deterministic and compartimental, in which the gastrointestinal tract (GIT) $\left(\mathrm{C}_{1}\right)$, plasma $\mathrm{C}_{2}$, bones $\left(C_{3}\right)$ and soft tissues (liver, heart, kidney and muscle) $\left(C_{4}\right)$ represented the compartments. Metabolism data and kinetics of $\mathrm{P}$ in tissues were obtained by the isotopic dilution technique. The simple superphosphate is the phosphorus source with the smallest capacity for polluting the environment, best bioavailability and most efficient use for the metabolism of growing swine.

Key Words: biomatematic model, endogenous phosphorus, isotopic dilution, metabolism, radiophosphorus

\section{Introdução}

A técnica de modelagem tornou-se importante no estudo da fisiologia digestiva de minerais, pela precisão com que simula e representa os fenômenos naturais e biológicos a partir de dados observados e obtidos em experimentação científica.
A utilização de dados obtidos com radionuclídeos permite estudar fenômenos complexos, com alta precisão, pois possibilita o acompanhamento passo a passo das reações e dos mecanismos biológicos ou naturais envolvidos e a quantificação das variáveis, de acordo com o interesse do pesquisador. Desta maneira, o resultado das pesquisas, ao ser extrapolado de forma matemática, para 
o universo da população estudada, torna a informação mais disponível e utilizável, podendo reduzir o tempo e os gastos com repetição de experimentos com objetivos similares.

No Brasil existem grandes reservas de rochas fosfáticas que podem ser utilizadas na nutrição animal, aproximadamente 2,9 bilhões de toneladas, e isto tem despertado o interesse de pesquisadores brasileiros em avaliar esses fosfatos como fontes alternativas em rações para suínos (Bellaver et al., 1984; Gomes et al., 1992; Curtarelli et al., 1989; Figueirêdo et al., 2001; Teixeira et al., 2004).

Um dos maiores problemas das fontes naturais de rochas fosfatadas está relacionado ao conteúdo de flúor. Em pequena proporção, esse mineral é desejável nas dietas para dar mais dureza e resistência aos dentes dos animais, porém, por ser um mineral tóxico e de efeito acumulativo, seu excesso pode inibir a glicólise e o ciclo de Krebs, afetando o desempenho dos animais (Moreira \& Miyada, 1998).

Todas as fontes naturais de rochas fosfatadas estudadas no Brasil têm apresentado baixos teores de flúor em comparação a fontes de outros países (Harmon et al., 1970) e níveis de metais pesados abaixo dos considerados normais pelos padrões internacionais (Barbosa et al., 1991; Teixeira, 2004).

O baixo teor de flúor é conseqüência da origem geológica dos fosfatos brasileiros, que é ígnea (Figueirêdo, 1998); os fosfatos de origem sedimentar, no entanto, contêm alto teor de flúor (Rosa, 1983). Outros fatores que requerem mais estudos são a composição química das rochas, que não é uniforme, e os níveis de metais pesados - até o fosfato bicálcico merece atenção especial (Junqueira, 1993).

A grande preocupação dos pesquisadores é fato que todo o fósforo não absorvido é excretado nos dejetos, que são utilizados como adubo orgânico.

O uso das fezes em adubação no solo de maneira indiscriminada pode provocar danos ao meio ambiente, quando os teores de fósforo excedem as exigências das plantas. O acúmulo do mineral no solo possibilita que parte se ligue às partículas do solo, tornando-se um contaminante em potencial para rios, lagos e córregos.

O objetivo nesta pesquisa foi avaliar a fisiologia digestiva de suínos alimentados com rações suplementadas com diversas fontes de fósforo relacionando a qualidade nutricional dessas fontes e a redução do impacto ambiental provocado pela excreção de fósforo no ambiente.

\section{Material e Métodos}

O experimento foi realizado no Laboratório de Nutrição Animal do Centro de Energia Nuclear na Agricultura da Universidade de São Paulo, em Piracicaba, São Paulo, utilizando-se 21 suínos mestiços, machos castrados com peso inicial de $31,94 \pm 2,88 \mathrm{~kg}$, distribuídos em delineamento de blocos casualizados, com sete dietas e três repetições. Foram utilizados como critérios para formação dos blocos o peso e a idade dos animais.

As dietas consistiram de uma ração basal composta de milho, farelo de soja e óleo vegetal (controle), suplementada com fosfato bicálcico (FBC), fosfato monobicálcio (MBC), superfosfato triplo (ST), superfosfato simples (SS), ácido fosfórico (AcF) ou fosfato de rocha catalão.

O experimento teve duração de 14 dias ( 7 de adaptação e 7 de coletas). No primeiro dia da fase experimental, cada animal recebeu, por via endovenosa, $0,5 \mathrm{~mL}$ de uma solução radioativa com $7,4 \mathrm{MBq}$ de ${ }^{32} \mathrm{P}$. A solução radioativa, preparada de acordo com Vitti et al. (2006), foi constituída de fosfato de sódio $\left(\mathrm{Na}_{2} \mathrm{H}^{32} \mathrm{PO}_{4}\right)$, livre de carregador, adquirida no Instituto de Pesquisas Energéticas e Nucleares (IPEN).

Nos três últimos dias da fase de adaptação foram coletadas amostras de fezes e urina para determinação do P inorgânico.

Após a aplicação do material radioativo, foram coletadas diariamente amostras de sangue, fezes e urina. No final do período experimental, os suínos foram sacrificados de acordo com aprovação da Comissão de Ética em Experimentação com animais (CEEA) do Centro de Energia Nuclear da Agricultura/CENA) e amostras de tecidos (osso, músculo (Longissimus dorsi), coração, fígado e rim) foram coletadas.

As amostras de sangue foram centrifugadas imediatamente após a coleta a $1.100 \mathrm{~g}$ durante 10 minutos e o plasma separado. Para detecção da radioatividade, $0,5 \mathrm{~mL}$ de plasma foi adicionado em água deionizada em frasco de contagem para determinação por efeito Cerenkov (Vitti et al., 2006). Ao volume de $1 \mathrm{~mL}$ de plasma foram adicionados $9 \mathrm{~mL}$ de ácido tricloroacético a $10 \%$ para precipitação de proteínas e o teor de fósforo inorgânico foi determinado por colorimetria (Fiske \& Subbarow, 1925).

As fezes foram coletadas durante sete dias, pesadas, maceradas e homogeneizadas. Alíquotas de $1 \mathrm{~g}$ foram colocadas em cadinhos de porcelana para determinação da matéria seca $\left(100^{\circ} \mathrm{C}\right)$ e das cinzas $\left(500^{\circ} \mathrm{C}\right)$. As cinzas foram digeridas com $10 \mathrm{~mL}$ de ácido sulfúrico $(18 \mathrm{~N})$ e o material digerido foi colocado em frascos de cintilação, completando-se o volume para $20 \mathrm{~mL}$ com água destilada para determinação da radioatividade por efeito Cerenkov. Cerca de $1 \mathrm{~g}$ de fezes foi digerido com $5 \mathrm{~mL}$ de ácido clorídrico concentrado e filtrado; ao volume de $2,5 \mathrm{~mL}$ do filtrado foram adicionados $2,5 \mathrm{~mL}$ de água deionizada e $2 \mathrm{~mL}$ de reagente misto (quantidades iguais de molibdato de amônia a 5\% e vanadato de amônia a $0,25 \%$ ). As leituras do fósforo inorgânico foram feitas por colorímetro (Sarruge \& Haag, 1974). 
Das amostras de urina coletadas, $0,5 \mathrm{~mL}$ foi diluído em 19,5 mL de água deionizada, e a atividade foi determinada por efeito Cerenkov. O fósforo inorgânico foi determinado após diluição de $1 \mathrm{~mL}$ de urina em $9 \mathrm{~mL}$ de ácido tricloroacético (10\%) (Fiske \& Subbarow, 1925)

$\mathrm{Na}$ fase de adaptação, as dietas experimentais foram distribuídas em duas refeições (pela manhã, às 10 h, e à tarde, às 18 h), com ração fornecida à vontade e registro do consumo.

Durante a fase de coleta, as dietas experimentais também foram distribuídas em duas refeições, porém com consumo diário baseado no peso metabólico $\left(\mathrm{W}^{0,75}\right)$, considerando o menor consumo, entre os animais de cada bloco, na fase de adaptação, com água fornecida à vontade.

Com exceção do fósforo da dieta controle, as dietas foram formuladas para atender às exigências nutricionais dos animais conforme recomendação de Rostagno et al. (2005) (Tabela 1).

O modelo matemático proposto o estudo do fluxo de fósforo em suínos (Figura 1) baseou-se nos estudos de
Fernandez (1995), Lopes et al. (1999, 2001) e Moreira et al. (2004), combinando dados dos estudos de metabolismo e cinética do fósforo nos tecidos.

O modelo foi determinístico e compartimental, de modo que os compartimentos C-1 trato gastrintestinal, C-2 plasma, C-3 ossos e C-4 tecidos moles e fígado, coração, rim e músculo foram estudados em conjunto, de acordo com a proporção dos seus pesos.

Os parâmetros foram estimados por meio de equações envolvendo as variáveis: $\mathrm{P}$ consumido $\left(\mathrm{V}_{\mathrm{I}}\right), \mathrm{P}$ total excretado nas fezes $\left(\mathrm{V}_{\mathrm{F}}\right)$, com as frações de origem alimentar $\left(\mathrm{V}_{\mathrm{FD}}\right)$ e endógena $\left(\mathrm{V}_{\mathrm{f}}\right)$, $\mathrm{P}$ excretado na urina $\left(\mathrm{V}_{\mathrm{U}}\right)$, absorção de $\mathrm{P}$ de origem alimentar $\left(\mathrm{V}_{\mathrm{aa}}\right)$ e reabsorção do fósforo de origem endógena $\left(\mathrm{V}_{\mathrm{af}}\right)$, valor da fração de $\mathrm{P}$ endógeno, proveniente de secreções e de células de descamação, que chegam ao trato gastrintestinal $\left(\mathrm{V}_{\mathrm{IT}}\right)$, absorção total de $\mathrm{P}$, incluindo as frações de origem endógena e alimentar $\left(\mathrm{V}_{\mathrm{aT}}\right)$ e balanço de $\mathrm{P}\left(\mathrm{B}_{\mathrm{a}}\right)$, definidas segundo Fernandez (1995) (equações $1 \mathrm{a} 6$ ). O valor do $\mathrm{P}$ proveniente do total absorvido $\left(\mathrm{V}_{\mathrm{aT}}\right)$ que retorna ao trato gastrintestinal $\left(\mathrm{V}_{\mathrm{eD} 1}\right)$ foi calculado

Tabela 1 - Composição das dietas experimentais

\begin{tabular}{|c|c|c|c|c|c|c|c|}
\hline \multirow[t]{2}{*}{ Ingrediente } & \multicolumn{7}{|c|}{ Fonte de fósforo } \\
\hline & Controle & $\begin{array}{l}\text { Fosfato } \\
\text { bicálcico }\end{array}$ & $\begin{array}{c}\text { Fosfato } \\
\text { monobicálcico }\end{array}$ & $\begin{array}{l}\text { Superfosfato } \\
\text { triplo }\end{array}$ & $\begin{array}{l}\text { Superfosfato } \\
\text { simples }\end{array}$ & $\begin{array}{l}\text { Ácido } \\
\text { fosfórico }\end{array}$ & $\begin{array}{c}\text { Fosfato Rocha } \\
\text { Catalão }\end{array}$ \\
\hline Farelo de soja & 26,30 & 26,30 & 26,30 & 26,3000 & 26,30 & 26,30 & 26,30 \\
\hline Milho & 68,00 & 68,00 & 68,00 & 68,00 & 68,00 & 68,00 & 68,00 \\
\hline L-lisina & 0,10 & 0,10 & 0,10 & 0,10 & 0,10 & 0,10 & 0,10 \\
\hline Óleo de soja & 1,65 & 1,65 & 1,65 & 1,65 & 1,65 & 1,65 & 1,65 \\
\hline Sal & 0,30 & 0,30 & 0,30 & 0,30 & 0,30 & 0,30 & 0,30 \\
\hline Mistura mineral $^{1}$ & 0,05 & 0,05 & 0,05 & 0,05 & 0,05 & 0,05 & 0,05 \\
\hline Mistura vitamínico ${ }^{1}$ & 0,10 & 0,10 & 0,10 & 0,10 & 0,10 & 0,10 & 0,10 \\
\hline Areia lavada & 1,78 & 1,23 & 1,15 & 1,06 & 0,0 & 0,70 & 1,62 \\
\hline Calcário & 1,72 & 0,99 & 1,17 & 1,28 & 0,36 & 1,78 & 0,00 \\
\hline Fosfato bicálcico ${ }^{3}$ & & 1,28 & & & & & \\
\hline Fosfato monobicálcico ${ }^{3}$ & & & 1,18 & & & & \\
\hline Superfosfato triplo ${ }^{3}$ & & & & 1,160 & & & \\
\hline Superfosfato simples ${ }^{3}$ & & & & & 3,14 & & \\
\hline Ácido fosfórico ${ }^{3}$ & & & & & & 1,02 & \\
\hline Fosfato rocha catalão ${ }^{3}$ & & & & & & & 1,88 \\
\hline Total & 100,00 & 100,00 & 100,00 & 100,00 & 100,00 & 100,00 & 100,00 \\
\hline \multicolumn{8}{|l|}{ Composição calculada } \\
\hline Energia digestível $\mathrm{kcal} / \mathrm{kg}$ & 3.400 & 3.400 & 3.400 & 3.400 & 3.400 & 3.400 & 3.400 \\
\hline Proteína bruta(\%) & 17,90 & 17,90 & 17,90 & 17,90 & 17,90 & 17,90 & 17,90 \\
\hline Fósforo total (\%) & 0,32 & 0,56 & 0,56 & 0,56 & 0,56 & 0,56 & 0,56 \\
\hline Cálcio (\%) & 0,78 & 0,78 & 0,78 & 0,78 & 0,78 & 0,78 & 0,78 \\
\hline Sódio (\%) & 0,16 & 0,16 & 0,16 & 0,16 & 0,16 & 0,16 & 0,16 \\
\hline Lisina total $(\%)$ & 0,98 & 0,98 & 0,98 & 0,98 & 0,98 & 0,98 & 0,98 \\
\hline Metionina $(\%)$ & 0,29 & 0,29 & 0,29 & 0,29 & 0,29 & 0,29 & 0,29 \\
\hline Metionina + cistina $(\%)$ & 0,59 & 0,59 & 0,59 & 0,59 & 0,59 & 0,59 & 0,59 \\
\hline Treonina (\%) & 0,69 & 0,69 & 0,69 & 0,69 & 0,69 & 0,69 & 0,69 \\
\hline Triptofano (\%) & 0,21 & 0,21 & 0,21 & 0,21 & 0,21 & 0,21 & 0,21 \\
\hline
\end{tabular}

${ }^{1}$ Conteúdo/kg: Fe - 100 g; Cu - 10 g; Co 1 g; 40g Mn; Zn - 100 g; I - 1,5 g; Se - 0,3 g; Excipiente, q.s.p.

${ }^{2}$ Conteúdo/kg: vit.A - 6.000.000 UI; vit. $\mathrm{D}_{3}-1.500 .000 \mathrm{UI}$; vit. E - 15.000 ; vit. $\mathrm{B}_{1}-1,35 \mathrm{~g}$; vit. $\mathrm{B}_{2}-4,0 \mathrm{~g}$; vit. $\mathrm{B}_{6}-2$ g; ácido pantotênico - $9,35 \mathrm{~g}$; vit. $\mathrm{K}_{3}-1,5 \mathrm{~g}$; ácido nicotínico - $20 \mathrm{~g}$; vit $\mathrm{B}_{12}-20,0 \mathrm{~g}$; ácido fólico - 0,6 g; biotina 0,08 - g; excipiente q.s.p. - $1.000 \mathrm{~g}$.

${ }^{3}$ Análise realizada no Laboratório de Nutrição Animal do Departamento de Zootecnia da UFV.

Conteúdo $(\%)$ de cálcio e fósforo das fontes: fosfato bicálcico $(23,25 ; 18,66)$; monobicálcico $(19,5 ; 20,29)$; superfosfato triplo $(16,46 ; 20,63)$; superfosfato simples $(17,2$;

$7,62)$; ácido fosfórico $(0,00 ; 19,68)$; e fosfato de rocha catalão $(36,00 ; 12,73)$. 


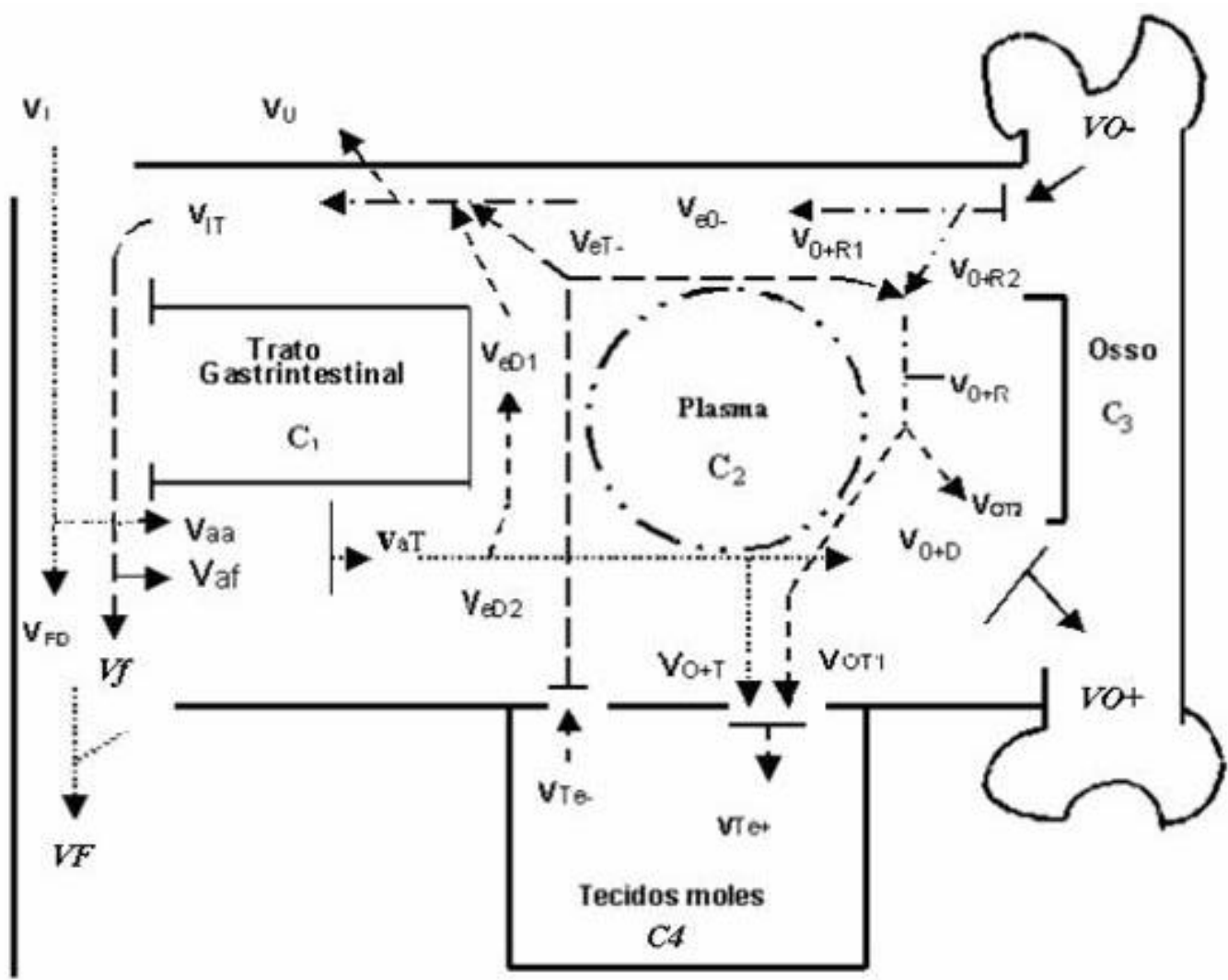

Figura 1 - Modelo do fluxo de P no metabolismo de suínos.

Legenda: VI - Quantidade de P ingerido, VF - perda total de P nas fezes, vf - P endógeno fecal, VDF - P nas fezes de origem dietética, VU - perda de P na urina, Vaa - P absorvido de origem alimentar, VIT - P de origem endógena que chega ao trato gastrointestinal - TGI, Vaf - P endógeno reabsorvido no TGI, VaT - total de P absorvido, VeD1 - P do VaT que retorna ao TGI, VeD2 - P do VaT distribuído para os ossos e tecidos moles, VO+D - P do VaT incorporado nos ossos, VO+ - P incorporado nos ossos, VO- - P reabsorvido dos ossos, VeO- - P reabsorvido dos ossos que retorna ao TGI, VO+R1 - P dos tecidos moles reciclado para os ossos e tecidos moles, VO+R2 - P dos ossos reciclado para os ossos e tecidos moles, VO+R - total de $\mathrm{P}$ reciclado para os ossos e tecidos moles, VOT1 - P do VO+R reincorporado nos tecidos moles, VOT2 - P_do VO+R reincorporado nos ossos, VTe+ $\mathrm{P}$ incorporado nos tecidos moles, VTe- - P reabsorvido dos tecidos moles, VeT- - P reabsorvido dos tecidos moles que retorna ao TGI.

pela diferença entre o $\mathrm{P}$ do $\mathrm{V}_{\mathrm{aT}}$ e o $\mathrm{P}$ incorporado $\mathrm{em}_{3} \mathrm{e}$ $\mathrm{C}_{4}$, após subtrair-se o $\mathrm{P}$ reciclado que retorna a estes compartimentos. O restante do total absorvido $\left(\mathrm{V}_{\mathrm{eD} 2}\right)$ foi distribuído proporcionalmente entre $\mathrm{C}_{3} \mathrm{eC}_{4}\left(\mathrm{~V}_{0+\mathrm{T}} \mathrm{e} \mathrm{V}_{0+\mathrm{D}}\right)$, de acordo com as equações 7 a 10 .

$\mathrm{V}_{\mathrm{FD}}=\mathrm{VF}-\mathrm{Vf}$

(equação 1)

$\mathrm{V}_{\mathrm{aa}}=\mathrm{V}_{\mathrm{I}}-\mathrm{V}_{\mathrm{FD}}$

(equação 2)

$\mathrm{V}_{\mathrm{IT}}=\mathrm{V}_{\mathrm{f}} /\left[1-\left(\mathrm{V}_{\mathrm{aa}} / \mathrm{V}_{\mathrm{I}}\right)\right]$

(equação 3)

$\mathrm{V}_{\mathrm{af}}=\mathrm{V}_{\mathrm{IT}}-\mathrm{V}_{\mathrm{f}}$

(equação 4)

$\mathrm{V}_{\mathrm{aT}}=\mathrm{V}_{\mathrm{aa}}+\mathrm{V}_{\mathrm{af}}$

(equação 5)

$\mathrm{B}_{\mathrm{a}}=\mathrm{V}_{\mathrm{I}}-\mathrm{V}_{\mathrm{F}}-\mathrm{V}_{\mathrm{U}}$

(equação 6)

$\mathrm{V}_{\mathrm{eD} 1}=\mathrm{V}_{\mathrm{aT}}-\left(\mathrm{V}_{\mathrm{O}+}-\mathrm{V}_{\mathrm{oR} 2}\right)-\left(\mathrm{V}_{\mathrm{Te}+}-\mathrm{V}_{\mathrm{O}+\mathrm{R} 1}\right) \quad($ equação 7$)$

$\mathrm{V}_{\mathrm{eD} 2}=\mathrm{V}_{\mathrm{aT}}-\mathrm{V}_{\mathrm{eD} 1}$
$\left[\mathrm{V}_{\mathrm{O}+} \mathrm{x}\left(\mathrm{V}_{\mathrm{aT}} \mathrm{x} \% \mathrm{P}\right.\right.$ incorporado no osso $\left.)\right]$

$$
\mathrm{V}_{\mathrm{O}+\mathrm{D}}=\frac{}{\left[\mathrm{V}_{\mathrm{o}-\mathrm{-}} \mathrm{x}\left(\mathrm{V}_{\mathrm{aT}} \mathrm{x} \% \mathrm{P} \text { incorporado no osso }\right)\right]}
$$

(equação9)

$$
\mathrm{V}_{\mathrm{O}+\mathrm{T}}=\frac{\left[\mathrm{V}_{\mathrm{Te}+} \times\left(\mathrm{V}_{\mathrm{aT}} \times \% \text { P incorporado nos tecidos }\right)\right]}{\left[\mathrm{V}_{\mathrm{Te}-} \mathrm{x}\left(\mathrm{V}_{\mathrm{aT}} \times \% \text { P incorporado nos tecidos }\right)\right]}
$$

(equação 10)

$\mathrm{Na}$ determinação do fósforo incorporado no osso $\left(\mathrm{V}_{0+}\right)$ e nos tecidos moles, fígado, coração, rim e músculo $\left(\mathrm{V}_{\mathrm{Te}+}\right)$, considerou-se a concentração de fósforo $(\mathrm{mg} / \mathrm{gMS})$. O percentual de ${ }^{32} \mathrm{P}[(\mathrm{cpm}$ amostra/g de MS $) /($ dose total injetada) $] /[(\mathrm{mgP} / \mathrm{g} \mathrm{MS})]$ e a atividade específica dos tecidos [(\% da atividade injetada de $\left.{ }^{32} \mathrm{P}\right) /(\mathrm{mgP} / \mathrm{gMS}$ do tecido $\left.)\right]$ foram calculados de acordo com Lopes (1998). Para o ${ }^{32} \mathrm{P}$ 
retido nos tecidos moles, foi considerada a média dos valores de cada tecido estudado. A quantidade de matéria seca (MS) de cada tecido foi determinada pelo produto entre o percentual de MS obtida e o peso dos tecidos, definidos segundo dados de pesquisa de Davidson (1966) e Lopes (1988). Foi utilizado ainda neste processo o decaimento físico (T1/2) e biológico ( $\mathrm{Tb})$ do ${ }^{32} \mathrm{P}$, fundamentado na International Atomic Energy Agency (1979), uma vez que os animais foram sacrificados uma semana após a aplicação do ${ }^{32} \mathrm{P}$ e a contagem do material radioativo efetuada oito dias após o abate.

$\mathrm{O}$ valor do ${ }^{32} \mathrm{P}$ retido ( $\mathrm{mg} / \mathrm{gMS}$ ) foi determinado pelo produto do percentual de ${ }^{32} \mathrm{P}$ retido e a concentração total de P nos compartimentos C-1 e C-3 (mg/gMS).

Após a correção do decaimento do ${ }^{32} \mathrm{P}$ para a data de sua introdução na corrente sanguínea, calculou-se o P incorporado (mg/g MS) nos compartimentos 3 e 4 pela relação entre o valor de ${ }^{32} \mathrm{P}$ corrigido e o da atividade específica em cada compartimento envolvido, e o $\mathrm{P}$ total incorporado ( $\mathrm{mg}$ ) nos compartimentos $\mathrm{C}_{3}$ e $\mathrm{C}_{4}$ foi obtido pelo produto entre a $\mathrm{MS}(\mathrm{g})$ do compartimento e o $\mathrm{P}$ incorporado (mg). Os valores de $\mathrm{V}_{0+}$ e $\mathrm{V}_{\mathrm{Te}+}(\mathrm{mgP} / \mathrm{kgPV}$. dia) foram obtidos pela relação entre o $P$ total incorporado no compartimento e o peso do animal $(\mathrm{g})$. As equações 11 a 24 caracterizam essas determinações.

${ }^{32} \mathrm{P}$ retido no osso $\left(\mathrm{mg}{ }^{32} \mathrm{P} / \mathrm{g} \mathrm{MS}\right)=\mathrm{P}$ inorgânico $(\mathrm{mg} \mathrm{P} / \mathrm{gMS}) \times{ }^{32} \mathrm{P}$ retido $(\%)$ (equação 11)

$\mathrm{P}$ total incorporado osso $(\mathrm{mg} \mathrm{P})=\mathrm{P}$ total osso $(\mathrm{mg} \mathrm{P} / \mathrm{g} \mathrm{MS})$ $\times$ MS osso (g MS)

(equação 12)

$\mathrm{P}$ total incorporado osso (mg P)

$\mathrm{Vo}+(\mathrm{mg} \mathrm{P} / \mathrm{kg}$ peso animal $)=-\times 1000$

Peso total do suíno $(\mathrm{kg})$ (equação 13)

Peso total tecidos $(\mathrm{g})=$ Peso suíno $(\mathrm{g}) \times \%$ Peso tecidos (equação 14)

MS tecidos $(\mathrm{g})=$ Peso total tecidos $(\mathrm{g}) \times \%$ MS tecidos (equação 15)

${ }^{32} \mathrm{P}$ retido fígado $\left(\mathrm{mg}{ }^{32} \mathrm{P} / \mathrm{g} \mathrm{MS}\right)=\mathrm{P}$ inorgânico $(\mathrm{mg} \mathrm{P} / \mathrm{g} \mathrm{MS}) \times \%{ }^{32} \mathrm{P}$ retido (equação 16)

${ }^{32} \mathrm{P}$ retido coração $\left(\mathrm{mg}{ }^{32} \mathrm{P} / \mathrm{g}\right.$ MS $)=\mathrm{P}$ inorgânico $(\mathrm{mg} \mathrm{P} / \mathrm{g}$ $\mathrm{MS}) \times \%{ }^{32} \mathrm{P}$ retido (equação 17)

${ }^{32} \mathrm{P}$ retido $\operatorname{rim}\left(\mathrm{mg}{ }^{32} \mathrm{P} / \mathrm{g} \mathrm{MS}\right)=\mathrm{P}$ inorgânico $(\mathrm{mg} \mathrm{P} / \mathrm{g} \mathrm{MS})$ $\times \%{ }^{32} \mathrm{P}$ retido

(equação 18)
${ }^{32} \mathrm{P}$ retido músculo $\left(\mathrm{mg}^{32} \mathrm{P} / \mathrm{g} \mathrm{MS}\right)=\mathrm{P}$ inorgânico $(\mathrm{mg} \mathrm{P} / \mathrm{g}$ $\mathrm{MS}) \times \%{ }^{32} \mathrm{P}$ retido (equação 19)

$\mathrm{P}$ total incorporado tecido $(\mathrm{mg} \mathrm{P})=\mathrm{P}$ total tecido $(\mathrm{mg} \mathrm{P} / \mathrm{g}$ MS) $\times$ MS tecido $(\mathrm{g})$

(equação 20)

P total incorporado tecido (mg P)

$\mathrm{V}_{\mathrm{Te}+}(\mathrm{mg} \mathrm{P} / \mathrm{kg}$ peso animal $)=\frac{}{\text { Peso do suíno }(\mathrm{kg})} \times 1000$ (equação 21)

$\mathrm{T} 1 / 2=0,693$ /constante de decaimento $(\mathrm{K}) \quad$ (equação 22$)$ Atividade do material radioativo no tempo " $t$ " $(\mathrm{A})=$ Atividade inicial $\left(\mathrm{A}_{\mathrm{o}}\right) \mathrm{x} \mathrm{e}^{-\mathrm{kt}}$ (equação 23)

$\mathrm{Tb}=0,693 \mathrm{t} / \ln \left(\mathrm{A}_{\mathrm{o}} / \mathrm{A}\right)$

(equação 24)

O fósforo reabsorvido nos compartimentos $\mathrm{C}-3\left(\mathrm{~V}_{0_{-}}\right)$ e C-4 ( $\left.\mathrm{V}_{\mathrm{Te}}\right)$ foi calculado deduzindo-se, respectivamente, de $\mathrm{V}_{0+}$ e de $\mathrm{V}_{\mathrm{Te+}}$ o valor do balanço de fósforo multiplicado pela proporção de fósforo incorporado no compartimento em estudo, equações 25 e 26.

Para determinação do fósforo reciclado que retorna aos compartimentos $\mathrm{C}-3$ e $\mathrm{C}-4\left(\mathrm{~V}_{0+\mathrm{R} 1}\right.$ e $\left.\mathrm{V}_{0+\mathrm{R} 2}\right)$ e ao trato gastrintestinal $\left(\mathrm{V}_{\mathrm{eo}}-\mathrm{V}_{\mathrm{eT}}\right)$, o cálculo foi realizado com base nas equações 27 a 30, segundo Fernandez (1995) e Lopes et al. (2001), adaptando-se ao modelo a proporção incorporada em cada um dos dois compartimentos, visto que neste trabalho o osso foi o único compartimento que mobilizou fósforo. $\mathrm{O}$ valor de fósforo proveniente do osso e dos tecidos moles reciclado para os tecidos moles $\left(\mathrm{V}_{0+\mathrm{R}}\right)$, o do fósforo do $\mathrm{V}_{0+\mathrm{R}}$ reincorporado nos tecidos moles $\left(\mathrm{V}_{\mathrm{TO} 1}\right)$ e o fósforo do $\mathrm{V}_{\mathrm{o}+\mathrm{R}}$ reincorporado nos ossos $\left(\mathrm{V}_{\mathrm{T} 02}\right)$ foram determinados de acordo com as equações 31 a 33 .

$\mathrm{V}_{\mathrm{O}-}=\mathrm{V}_{0+}-($ BA X \% P incorporado no osso) (equação 25)

$\mathrm{V}_{\mathrm{TE}-}=\mathrm{V}_{\mathrm{TE}+}-($ BA X \% P incorporado tecido) (equação 26)

$\mathrm{V}_{\mathrm{Te}+} \times \mathrm{V}_{\mathrm{Te}-} \mathrm{V}_{0+\mathrm{R} 1}=\left[\mathrm{V}_{\mathrm{TE}+}\left(\mathrm{V}_{\mathrm{AT}} \mathrm{X} \%\right.\right.$ P incorporado tecido $\left.)\right]$ (equação 27)

$\mathrm{V}_{\mathrm{o}+} \times \mathrm{V}_{\mathrm{o}-} \mathrm{V}_{0+\mathrm{R} 2}=\mathrm{V}_{\mathrm{o}-}+\left(\mathrm{V}_{\mathrm{aT}} \times \% \mathrm{P}\right.$ incorporado osso $)$ (equação 28)

$\mathrm{V}_{\mathrm{eo}-}=\mathrm{V}_{\mathrm{o}-}-\mathrm{V}_{\mathrm{o}+\mathrm{R} 2}$ (equação 29)

$\mathrm{V}_{\mathrm{eT}-}=\mathrm{V}_{\mathrm{Te}-}-\mathrm{V}_{\mathrm{oR} 1}$ (equação 30)

$\mathrm{V}_{\mathrm{o}+\mathrm{R}}=\mathrm{V}_{\mathrm{o}+\mathrm{R} 1}+\mathrm{V}_{\mathrm{o}+\mathrm{R} 2}$ (equação 31)

$\mathrm{V}_{\mathrm{To} 1}=\mathrm{V}_{\mathrm{O}+\mathrm{R}}-\left(\mathrm{V}_{\mathrm{o}+}-\mathrm{V}_{\mathrm{o}+\mathrm{D}}\right)$ (equação 32)

$\mathrm{V}_{\mathrm{To} 2}=\mathrm{V}_{\mathrm{O}+\mathrm{R}}-\mathrm{V}_{\mathrm{To} 1}$ 
A biodisponibilidade das dietas foi determinada pela relação entre o fósforo absorvido de origem alimentar $\left(\mathrm{V}_{\mathrm{aa}}\right)$ e o fósforo consumido $\left(\mathrm{V}_{\mathrm{I}}\right)$ (Teixeira et al., 2004).

A variáveis do modelo foram submetidas à análise da variância e ao teste Duncan, segundo procedimentos do Statistical Analysis System (SAS, 2000).

\section{Resultados e Discussão}

As fontes de fósforo não interferiram $(\mathrm{P}>0,05)$ nos consumos de ração e de fósforo (Tabela 2). Esse resultado era esperado, pois em ensaio de metabolismo o consumo é padronizado e a duração do período experimental reduzida. Entretanto, o consumo de fósforo da ração controle foi inferior ao das demais $(\mathrm{P}<0,05)$, uma vez que a ração controle não foi suplementada com fósforo inorgânico.

Quando o consumo de ração não é padronizado, a ingestão de fósforo está diretamente relacionada ao seu nível dietético na ração (Fernandez, 1995; Lopes, 1998; Figueirêdo et al., 2001) e tende a reduzir o consumo de ração, quando deficientes em fósforo. Contudo, resultados diferentes foram observados por Bellaver et al. (1983), que não verificaram diferença no consumo entre rações com ou sem fósforo suplementar.

A excreção fecal de origem alimentar foi menor nos animais alimentados com a ração controle em comparação àquelas suplementadas com fontes de fósforo (Tabela 3 ). Quando avaliadas as relações entre a excreção fecal de origem alimentar $\left(\mathrm{V}_{\mathrm{FD}}\right)$ e a quantidade de fósforo consumido
$\left(\mathrm{V}_{\mathrm{I}}\right)$, os valores não apresentaram diferenças estatísticas ( $P>0,05)$. Contudo, diferiram entre as fontes de fósforo: 0,50 , controle; 0,41 , fosfato bicálcico; 0,39 , fosfato monobicálcico; 0,41 , superfosfato triplo; 0,36 , superfosfato simples; 0,38, ácido fosfórico; e 0,50, fosfato de rocha catalão. Para cada 1,00 g de $\mathrm{P}$ consumido nas rações controle e com fosfato de rocha catalão, a excreção nas fezes foi de 0,50 g. Entre as fontes estudadas, o superfosfato simples apresentou a melhor relação entre o fósforo consumido e excretado e, para cada $1 \mathrm{~g}$ de fósforo consumido, $0,36 \mathrm{~g}$ foram excretados, com diferença de $28,00 \%$ em relação a essas duas dietas.

O consumo de alimentos deficientes em fósforo pode provocar maior reabsorção óssea, promover elevação temporária da concentração de fósforo no plasma e aumentar as perdas endógenas e urinárias. Neste experimento não se observaram diferenças estatísticas para os níveis de fósforo reabsorvido do osso $\left(\mathrm{V}_{\mathrm{O}_{-}}\right)(\mathrm{P}>0,05)$, mas as dietas controle (sem fósforo inorgânico) e com fosfato de rocha catalão foram inferiores aos obtidos com as demais dietas. Estas observações confirmam estudos de Fernandez (1995), que observou comportamento similar entre níveis altos e médios de fósforo (334,29 e $220 \mathrm{mg} / \mathrm{kg}$ PV.dia), e Lopes et al. (2001), que observaram para o fósforo reabsorvido do osso $\left(\mathrm{V}_{\mathrm{o}_{-}}\right)$valor maior para a dieta controle $(0,30 \% \mathrm{P}$ total $)$ em comparação aos fosfatos de rocha Tapira, Patos de Minas e supertriplo $(0,56 \%$ de $\mathrm{P}$ total).

As excreções endógenas e fecais, de acordo com Breves \& Schroder (1991), representam uma rota de excreção

Tabela 2 - Fisiologia digestiva de suínos alimentados com rações com diferentes fontes de fósforo

\begin{tabular}{|c|c|c|c|c|c|c|c|c|}
\hline \multirow{2}{*}{$\begin{array}{l}\text { Parâmetro } \\
\text { (mg/kg PV.dia) }\end{array}$} & \multicolumn{7}{|c|}{ Fonte de fósforo } & \multirow[t]{2}{*}{ EPM } \\
\hline & Controle & $\begin{array}{l}\text { Fosfato } \\
\text { bicálcico }\end{array}$ & $\begin{array}{c}\text { Fosfato } \\
\text { monobicálcico }\end{array}$ & $\begin{array}{l}\text { Superfosfato } \\
\text { triplo }\end{array}$ & $\begin{array}{c}\text { Superfosfato } \\
\text { simples }\end{array}$ & $\begin{array}{l}\text { Ácido } \\
\text { fosfórico }\end{array}$ & $\begin{array}{c}\text { Fosfato Rocha } \\
\text { Catalão }\end{array}$ & \\
\hline Consumo de ração (g/kg PV.dia) & 35,55 & 35,10 & 34,67 & 34,90 & 35,51 & 34,85 & 34,84 & 0,85 \\
\hline $\mathrm{P}$ consumido $\left(\mathrm{V}_{\mathrm{I}}\right) *$ & $123,44 b$ & $224,63 \mathrm{a}$ & $221,90 \mathrm{a}$ & $223,34 \mathrm{a}$ & $227,27 \mathrm{a}$ & $223,07 \mathrm{a}$ & $222,96 a$ & 7,97 \\
\hline $\mathrm{P}$ endógeno para o TGI $\left(\mathrm{V}_{\mathrm{IT}}\right)$ & 15,40 & 30,33 & 22,46 & 25,63 & 21,60 & 27,67 & 13,57 & 10,12 \\
\hline $\mathrm{P}$ urina $\left(\mathrm{V}_{\mathrm{U}}\right) *$ & $0,32 \mathrm{c}$ & $2,25 \mathrm{bc}$ & $0,65 \mathrm{bc}$ & $5,82 \mathrm{abc}$ & $6,89 \mathrm{abc}$ & $10,89 \mathrm{a}$ & $0,86 \mathrm{bc}$ & 4,86 \\
\hline $\mathrm{P}$ endógeno fecal & 7,22 & 11,11 & 8,88 & 10,40 & 7,56 & 9,48 & 6,30 & 3,03 \\
\hline$P$ fecal de origem dietética (VFD) & 61,06 & 91,80 & 87,29 & 90,66 & 82,78 & 83,46 & 111,41 & 23,61 \\
\hline Relação $V_{\mathrm{FD}} / \mathrm{V}_{\mathrm{I}}$ & 0,50 & 0,41 & 0,39 & 0,41 & 0,36 & 0,38 & 0,50 & 0,11 \\
\hline $\mathrm{P}$ total nas fezes $\left(\mathrm{V}_{\mathrm{F}}\right)$ & 68,28 & 102,91 & 96,98 & 101,06 & 90,34 & 92,95 & 117,70 & 23,61 \\
\hline $\mathrm{P}$ dietético absorvido $\mathrm{V}_{\text {aa* }}$ & $62,37 \mathrm{~b}$ & $132,83 b$ & $134,60 b$ & $132,69 b$ & $144,43 \mathrm{a}$ & $139,61 \mathrm{a}$ & $111,55 \mathrm{ab}$ & 26,66 \\
\hline Disponibilidade biológica (\%) & 49,35 & 58,81 & 60,81 & 59,56 & 63,99 & 62,37 & 49,92 & 12,03 \\
\hline $\mathrm{P}$ endógeno reabsorvido & 8,19 & 19,22 & 13,58 & 15,22 & 14,04 & 18,19 & 7,30 & 8,27 \\
\hline Total de $\mathrm{P}$ absorvido $\left(\mathrm{V}_{\mathrm{aT}}\right)^{*}$ & $70,56 \mathrm{a}$ & $152,06 \mathrm{~b}$ & $148,18 b$ & $147,91 \mathrm{~b}$ & $158,53 b$ & $157,80 \mathrm{~b}$ & $118,86 \mathrm{ab}$ & 36,61 \\
\hline $\mathrm{P}$ incorporado no osso $\left(\mathrm{V}_{\mathrm{o}+}\right) *$ & $105,00 \mathrm{~b}$ & $123,46 \mathrm{ab}$ & $128,21 \mathrm{ab}$ & $131,82 \mathrm{a}$ & $132,72 \mathrm{a}$ & $129,16 \mathrm{ab}$ & $122,87 \mathrm{ab}$ & 8,64 \\
\hline $\mathrm{P}$ reabsorvido do osso $\left(\mathrm{V}_{\mathrm{o}_{-}}\right)$ & 61,83 & 23,41 & 26,79 & 31,54 & 24,41 & 33,53 & 36,36 & 21,41 \\
\hline Balanço de $\mathrm{P}$ no osso* & $43,16 \mathrm{a}$ & $87,60 \mathrm{ab}$ & $101,42 b$ & $100,28 b$ & $108,31 \mathrm{~b}$ & $95,63 b$ & $86,52 \mathrm{ab}$ & 19,80 \\
\hline $\mathrm{V}_{\mathrm{O} \text { - }}$ para o osso e tecidos moles & 55,40 & 20,29 & 23,32 & 27,08 & 20,89 & 27,13 & 33,77 & 18,28 \\
\hline Vo- para o TGI e urina $\left(\mathrm{V}_{\mathrm{eo}^{-}}\right)$ & 6,43 & 3,12 & 3,47 & 4,47 & 3,52 & 6,40 & 2,59 & 3,28 \\
\hline
\end{tabular}

*Médias seguidas de letras distintas na linha diferem $(\mathrm{P}<0,05)$ entre si pelo teste Duncan.

$\mathrm{EPM}=$ erro-padrão da média. 
Tabela 3 - Fisiologia digestiva de suínos alimentados com rações com diferentes fontes de fósforo

\begin{tabular}{|c|c|c|c|c|c|c|c|c|}
\hline \multirow{2}{*}{$\begin{array}{l}\text { Parâmetro } \\
\text { (mg/kg PV.dia) }\end{array}$} & \multicolumn{7}{|c|}{ Fonte de fósforo } & \multirow[t]{2}{*}{ EPM } \\
\hline & Controle & $\begin{array}{l}\text { Fosfato } \\
\text { bicálcico }\end{array}$ & $\begin{array}{c}\text { Fosfato } \\
\text { monobicálcico }\end{array}$ & $\begin{array}{l}\text { Superfosfato } \\
\text { triplo }\end{array}$ & $\begin{array}{c}\text { Superfosfato } \\
\text { simples }\end{array}$ & $\begin{array}{l}\text { Ácido } \\
\text { fosfórico }\end{array}$ & $\begin{array}{l}\text { Fosfato roch } \\
\text { catalão }\end{array}$ & \\
\hline $\mathrm{P}$ do $\mathrm{V}_{\mathrm{aT}}$ incorporado no osso $\left(\mathrm{V}_{\mathrm{o}+\mathrm{D}}\right) *$ & $49,59 \mathrm{~b}$ & $103,17 \mathrm{ab}$ & $102,03 \mathrm{ab}$ & $104,75 \mathrm{ab}$ & $111,83 \mathrm{~b}$ & $102,00 \mathrm{ab}$ & $89,00 \mathrm{ab}$ & 20,53 \\
\hline $\mathrm{P}$ incorporado nos tecidos $\left(\mathrm{V}_{\mathrm{Te}+}\right)$ & 28,58 & 24,70 & 30,21 & 23,41 & 26,74 & 31,23 & 25,32 & 8,96 \\
\hline $\mathrm{P}$ reabsorvido dos tecidos $\left(\mathrm{V}_{\mathrm{Te}}\right)$ & 16,91 & 5,18 & 6,55 & 7,23 & 5,01 & 7,42 & 7,44 & 4,95 \\
\hline Balanço de $\mathrm{P}$ nos tecidos & 11,67 & 19,42 & 23,65 & 16,19 & 21,73 & 23,60 & 17,88 & 8,35 \\
\hline $\mathrm{V}_{\mathrm{Te}-}$ para o osso e tecidos $\left(\mathrm{V}_{\mathrm{O}+\mathrm{R} 1}\right)$ & 15,25 & 7,08 & 18,02 & 6,33 & 4,29 & 5,91 & 6,91 & 9,34 \\
\hline $\mathrm{V}_{\mathrm{O}+\mathrm{R} 1}$ e $\mathrm{V}_{\mathrm{O}+\mathrm{R} 2}$ para osso e tecidos $\left(\mathrm{V}_{\mathrm{O}+\mathrm{R}}\right)$ & 70,75 & 24,86 & 29,01 & 33,41 & 25,18 & 33,14 & 40,68 & 22,39 \\
\hline $\mathrm{V}_{\mathrm{Te}}$ para o TGI e urina $(\mathrm{VeT}-)$ & 1,66 & 0,71 & 0,86 & 0,90 & 0,72 & 1,42 & 0,54 & 0,84 \\
\hline $\mathrm{P}$ do $\mathrm{V}_{\mathrm{aT}}$ para os tecidos moles $\left(\mathrm{V}_{\mathrm{o}+\mathrm{T}}\right)$ & 13,33 & 20,13 & 24,52 & 17,08 & 22,45 & 25,02 & 18,41 & 8,23 \\
\hline $\mathrm{V}_{\mathrm{O}+\mathrm{R}}$ reincorporado nos tecidos $\left(\mathrm{V}_{\mathrm{OT} 1}\right)$ & 15,25 & 4,57 & 5,69 & 6,33 & 4,29 & 6,01 & 7,15 & 4,25 \\
\hline $\mathrm{V}_{\mathrm{O}+\mathrm{R}}$ reincorporado no osso $\left(\mathrm{V}_{\mathrm{OT} 2}\right)$ & 55,40 & 22,17 & 23,32 & 27,08 & 21,15 & 27,13 & 33,77 & 17,57 \\
\hline $\mathrm{P}$ do $\mathrm{V}_{\mathrm{aT}}$ para TGI $\left(\mathrm{V}_{\mathrm{eD1}}\right)$ & 7,63 & 28,76 & 18,78 & 26,08 & 24,25 & 30,75 & 11,34 & 11,26 \\
\hline $\mathrm{P}$ do $\mathrm{V}_{\mathrm{aT}}$ para os ossos e tec. moles $\left(\mathrm{V}_{\mathrm{eD} 2}\right)$ & 62,93 & 123,03 & 129,40 & 121,83 & 134,28 & 127,05 & 107,52 & 25,18 \\
\hline
\end{tabular}

*Médias seguidas de letras distintas na linha diferem $(\mathrm{P}<0,05)$ entre si pelo teste Duncan.

$E P M=$ erro-padrão da média.

metabólica importante para o controle homeostático do fósforo no plasma em animais monogástricos. Neste experimento, os valores da variável $\mathrm{V}_{\mathrm{IT}}$, que representa também uma importante rota de excreção metabólica de fósforo, indicam relação similar com o fósforo dietético absorvido $\left(\mathrm{V}_{\mathrm{aa}}\right)$ das fontes estudadas (Figura 2), promovendo menor perda para as dietas controle e com fosfato de rocha catalão. Com base nestes dados, pode-se afirmar que, quanto maior a disponibilidade de fósforo, maior o retorno do fósforo via $\mathrm{V}_{\mathrm{IT}}$.

O fósforo endógeno reabsorvido no trato gastrintestinal representa a fração que retorna para a corrente sanguínea. Existe relação de similaridade com a variável $\mathrm{V}_{\mathrm{IT}}$, desta maneira, assim como ocorrem as perdas obrigatórias mínimas, também ocorre a absorção obrigatória mínima do fósforo endógeno excretado no trato digestivo (Figura 3).

Lopes et al. (2001) estudaram o modelo do fluxo biológico do fósforo de fontes de fosfato (fosfato bicálcico, Tapira, Patos de Minas, monoamônio, supertriplo e dieta controle) em suínos usando o ${ }^{32} \mathrm{P}$ como marcador e observaram os seguintes valores para a variável $\mathrm{V}_{\mathrm{IT}}: 16,69 ; 10,67 ; 9,99$; 16,$70 ; 9,85$ e $13,59 \mathrm{mg} / \mathrm{kgPV}$; e para o $\mathrm{V}_{\mathrm{af}}$ foram observados os valores: 10,$46 ; 5,96 ; 5,27 ; 11,00 ; 4,80$ e5,70 mg/kgPV, respectivamente, que expressam relação de similaridade. No estudo do fluxo biológico do fósforo no metabolismo de suínos alimentados com rações com diferentes fontes de fósforo, Moreira et al. (2002) também observaram relação similar quando estudaram as duas variáveis. De acordo com estas observações, pode-se afirmar que o aumento na excreção do fósforo endógeno para o trato gastrintestinal $\left(\mathrm{V}_{\mathrm{IT}}\right)$ é seguido pelo aumento no fósforo endógeno reabsorvido para o plasma. Isto diminui o impacto provocado pelas dietas deficientes em fósforo no metabolismo dos animais.

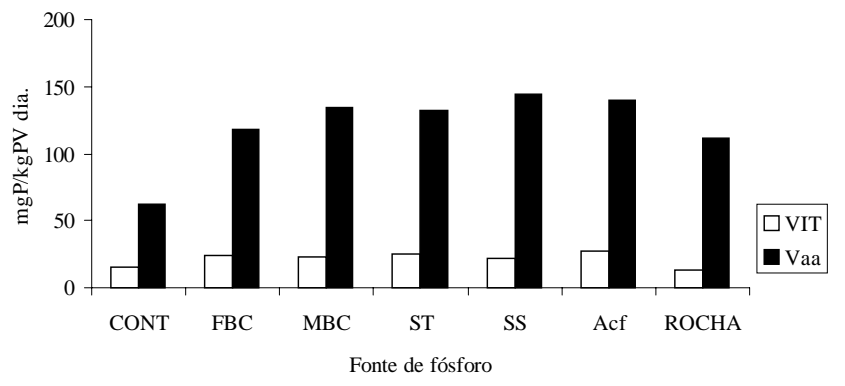

Figura 2 - Relação entre o P que retorna ao trato gastrointestinal (VIT) e o P dietético absorvido (Vaa).

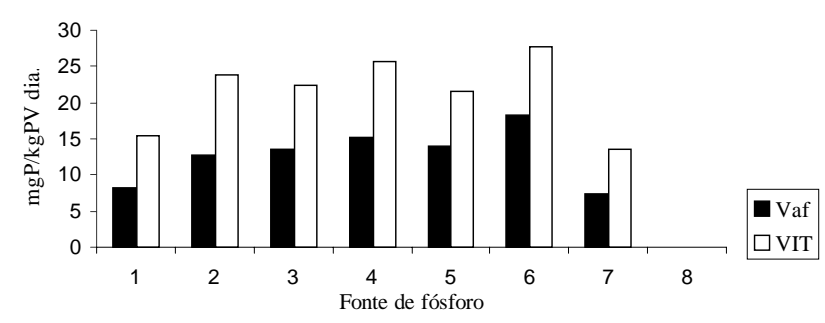

Figura 3 - Relação entre o P endógeno que retorna à corrente sanguínea (Vaf) e o P que retorna ao trato gastrointestinal (VIT).

As fontes de fósforo estudadas não influenciaram os valores de fósforo endógeno fecal (P>0,05), o que está de acordo com o descrito por Broce (1986) e Figueirêdo et al. (2001), que também observaram valores semelhantes entre as fontes de fósforo. Neste experimento, observou-se valor médio de $8,95 \mathrm{mg} / \mathrm{kg}$ PV.dia para as fontes suplementares estudadas, o que está de acordo com Cupák et al. (1972) e Jongbloeb et al. (1992), que relataram que as excreções 
endógenas de fósforo podem variar de 5 a $10 \mathrm{mg} / \mathrm{kg}$ PV.dia. Entretanto, as dietas com fosfato de rocha catalão, controle e com superfosfato simples apresentaram menores valores. Fernandez (1995) e Lopes et al. (1999) também observaram aumento nas perdas endógenas de fósforo com o aumento do nível de fósforo da ração.

As perdas urinárias foram afetadas $(\mathrm{P}<0,05)$ pelas fontes de fósforo, os menores valores foram obtidos para as dietas controle e com fosfatos monobicálcico, rocha catalão e bicálcico e os maiores valores para ácido fosfórico, superfosfato simples e superfosfato triplo. As maiores excreções urinárias sugerem que a absorção de fósforo dietético pode ter excedido as necessidades metabólicas dos animais, diminuindo proporcionalmente a incorporação do fósforo nos ossos $\left(\mathrm{V}_{\mathrm{o}+}\right)$ e tecidos $\left(\mathrm{V}_{\mathrm{Te}+}\right)$.

Não houve diferença estatística $(\mathrm{P}>0,05)$ entre as fontes estudadas para o fósforo excretado nas fezes, entretanto, a menor excreção ocorreu para as rações controle e com superfosfato simples e o maior, para a ração com fosfato de rocha catalão.

O superfosfato simples (SS) apresentou a melhor biodisponibilidade, pois foi metabolizado com maior eficiência pelo organismo dos animais, resultando em menor excreção nas fezes, e o fosfato de rocha catalão apresentou resultados opostos, resultando em maior excreção de fósforo nas fezes. A dieta controle (sem suplementação de $\mathrm{P}$ inorgânico) apresentou valores menores para fósforo nas fezes, em virtude da menor ingestão de fósforo pelos animais.

As fontes de fósforo influenciaram $(\mathrm{P}<0,05)$ os valores de fósforo incorporado no osso $\left(\mathrm{V}_{\mathrm{O}+}\right)$ e o balanço de fósforo osso. $\mathrm{O} \mathrm{V}_{\mathrm{aT}}$ representa a absorção total de fósforo, ou seja, o somatório do $\mathrm{V}_{\mathrm{aa}} \mathrm{e}_{\mathrm{af}}$. Os valores foram maiores para superfosfato simples e ácido fosfórico, o que está de acordo com os relatos de Teixeira et al. (2004), que estudaram as mesmas fontes de fósforo em experimento de biodisponibidade e observaram maiores valores para absorção verdadeira de fósforo, também para as fontes superfosfato simples e ácido fosfórico.

Os animais que se alimentaram com rações suplementadas com superfosfato simples e ácido fosfórico também apresentaram maiores valores $(\mathrm{P}<0,05)$ de $\mathrm{P}$ incorporado no osso $\left(\mathrm{V}_{\mathrm{o}+}\right)$, balanço de fósforo no osso e biodisponibilidade. A biodisponibilidade foi inferior à observada por Teixeira et al. (2004) em estudo com as mesmas fontes.

A dieta que não foi suplementada com fósforo apresentou biodisponibilidade de 49,35\%, semelhante aos valores de 49,00\%, encontrado por Figueiredo et al. (2001), e 43,82\%, obtido por Teixeira et al. (2004). A maior parte do fósforo dos vegetais (45 a 86\%), segundo Rostagno et al. (1987) encontra-se complexada nas moléculas de fitato, indisponível para animais monogástricos. De acordo com o NRC (1994), apenas 30\% do fósforo fítico está disponível, assim, as dietas podem ser formuladas utilizando-se esse valor como referência.

A biodisponibilidade do fosfato bicálcico, de 58,81\% obtida neste experimento assemelha-se aos resultados de 59,$61 ; 59,49 ; 56,90$ e $57,14 \%$ obtidos por Bellaver (1984), Lopes (1999a,b) e Figueiredo et al. (2001), respectivamente, em pesquisa com dietas e metodologias semelhantes. A fração do fósforo total absorvido $\mathrm{V}_{\mathrm{aT}} \mathrm{e}$ incorporado no osso $\left(\mathrm{V}_{\mathrm{o}+\mathrm{D}}\right)$ apresentou relação de similaridade $(\mathrm{P}<0,05)$ com o $\mathrm{V}_{\mathrm{aT}}$ : os maiores valores foram observados para o fosfato bicálcico, o monoamônio, o superfosfato simples, supertriplo e ácido fosfórico e os menores, para as dietas controle e com fosfato de rocha catalão. O fósforo total absorvido $\left(\mathrm{V}_{\mathrm{AT}}\right)$ está diretamente relacionado ao fósforo do $\mathrm{V}_{\mathrm{AT}}$ incorporado no osso $\left(\mathrm{V}_{\mathrm{O}+\mathrm{D}}\right)$, assim, quando aumenta o $\mathrm{V}_{\mathrm{AT}}$ também aumenta o $\mathrm{V}_{\mathrm{O}+\mathrm{D}}$.

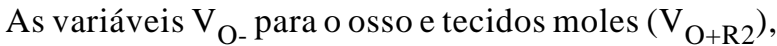
$\mathrm{V}_{\mathrm{O}-\text { para o }}$ TGI e urina $\left(\mathrm{V}_{\mathrm{eo}-}\right), \mathrm{P}$ incorporado nos tecidos $\mathrm{V}_{\mathrm{Te}+}$, $\mathrm{P}$ reabsorvido dos tecidos $\mathrm{V}_{\mathrm{Te}-}$, balanço de $\mathrm{P}$ nos tecidos, $V_{\text {te- }}$ para os ossos e tecidos moles $\left(V_{0+R 1}\right),\left(V_{0+R 1}\right)$ e $\mathrm{V}_{\mathrm{O}+\mathrm{R} 2}$ para o osso e tecidos $\left(\mathrm{V}_{\mathrm{O}+\mathrm{R}}\right), \mathrm{V}_{\mathrm{Te}}$ para o TGI e urina $\left(\mathrm{V}_{\mathrm{eT}-}\right)$, $\mathrm{P}$ do $\mathrm{V}_{\mathrm{aT}}$ para os tecidos moles $\left(\mathrm{V}_{\mathrm{O}+\mathrm{T}}\right), \mathrm{V}_{\mathrm{O}+\mathrm{R}}$ reincorporado nos tecidos $\left(\mathrm{V}_{\mathrm{OT} 1}\right), \mathrm{V}_{\mathrm{O}+\mathrm{R}}$ reincorporado no osso $\left(\mathrm{V}_{\mathrm{OT} 2}\right)$, P do $\mathrm{V}_{\mathrm{aT}}$ que retorna ao TGI $\left(\mathrm{V}_{\mathrm{eD} 1}\right)$ e $\mathrm{P}$ do $\mathrm{V}_{\mathrm{aT}}$ para os ossos e tecidos moles $\left(\mathrm{V}_{\mathrm{eD} 2}\right)$ não foram influenciados $(\mathrm{P}>0,05)$ pelas fontes de fósforo avaliadas.

\section{Conclusões}

O superfosfato simples destacou-se entre as fontes estudadas por sua menor poluição ao meio ambiente, melhor biodisponibilidade e pela maior eficiência de metabolização de suínos em crescimento. O fósforo endógeno que retorna ao trato gastrintestinal representa uma importante rota de excreção metabólica de fósforo e, quanto maior sua disponibilidade, maior seu retorno via $\mathrm{V}_{\mathrm{IT}}$. A fração do fósforo endógeno que retorna para a corrente sanguínea apresenta comportamento similar ao $\mathrm{V}_{\mathrm{IT}}$, confirmando que, da mesma maneira como ocorrem as perdas obrigatórias mínimas, também ocorrem a absorção obrigatória mínima do fósforo endógeno excretado no trato digestivo. Em dietas deficientes em fósforo, como a dieta controle, o impacto no metabolismo dos animais é reduzido pelo mecanismo de reabsorção do fósforo endógeno. 


\section{Literatura Citada}

BARBOSA, H.P.; MORAES, N.; FIALHO, E.T. et al. Desempenho produtivo e reprodutivo de suínos alimentados com fosfatos não convencionais. In MINI-SIMPÓSIO DO COLÉGIO BRASILEIRO DE NUTRIÇÃO ANIMAL, 1991, Campinas. Anais... Campinas: Colégio Brasileiro de Nutrição Animal, 1991. p.97-120.

BELlaVER, C.; GOMES, P.C.; SANTOS, D.L. Absorção e disponibilidade de fósforo para suínos, baseada na diluição isotópica $\left({ }^{32} \mathrm{P}\right)$. Pesquisa Agropecuária Brasileira, v.9, n.18, p.1053-1057, 1983.

BELlAVER, C.; GOMES, P.C.; FIALHO, D.L. Absorção e digestibilidade do fósforo dos fosfatos naturais em rações para suínos. Pesquisa Agropecuária Brasileira, v.19, n.12, p.1513-1518, 1984.

BREVES, G.; SCHRODER, B. Comparative aspects of gastrointestinal phosphorus metabolism. Nutrition Research Reviews, v.4, p.125-140, 1991.

BROCE, C.S. Comparação entre diferentes fontes de fósforo em rações para suínos nas fases de crescimento e terminação. 1986. 67f. Dissertação (Mestrado em Zootecnia) - Universidade Federal de Santa Maria, Santa Maria, 1986.

CUPÁK, M.; PROCHASKA, Z.; JAMBOR, V. Utilization of phosphorus compounds in pigs after endogenous phosphorus determination means of ${ }^{32} \mathrm{P}$. Acta Veterinaria Brno, v.41, p.257-262, 1972

CURTARELLI, S.M.; KRONKA, R.N.; PINHEIRO, M.G. et al Fosfato naturais em rações para suínos em crescimento e acabamento e seus efeitos no desenvolvimento e níveis de cálcio e fósforo no metacarpo. Ciência Zootécnica, v.4, p.14-15, 1989.

DAVIDSON, H.R. Pig meat: the carcass in relation to the live pig. In: DAVIDSON, H.R. (Ed.) The production and marketing of pigs. 3.ed. London: Longman, 1966. p.89-102.

FERNANDEZ, J.A. Calcium and phosphorus metabolism in growing pigs. III. A model resolution. Livestock Prodution Science, v.41, n.1, p.2255-2261, 1995.

FIGUEIRÊDO, A.V. Disponibilidade biológica de fósforo de cinco fosfatos, determinada com suínos em crescimento, através da técnica de diluição isotópica. 1998. 103f. Tese (Doutorado em Energia Nuclear na Agricultura) - Centro de Energia Nuclear na Agricultura, Universidade de São Paulo, Piracicaba, 1998

FIGUEIRÊDO, A.V.; VITTI, D.M.S.S.; LOPES, J.B. Disponibilidade biológica do fósforo de fontes fosfatadas determinada por intermédio da técnica de diluição isotópica para suínos em crescimento. Revista Brasileira de Zootecnia, v.30, n.5, p.1514-1520, 2001.

FISKE, C.H.; SUBBARROW, Y. The colorimetric determination of phosphorus. Journal Biological Chemistry, v.66, p.375$400,1925$.

GOMES, P.C.; LIMA, G.J.M.M.; GOMES, J.M.M. et al. Disponibilidade de fósforo nos fosfato de tapira, fosforíndus e na farinha de ossos para suínos. Revista da Sociedade Brasileira de Zootecnia, v.21, n.1, p.83-89, 1992.

HARMON, B.G.; SIMON, J.; BECKER, D.E. et al. Effect of source level of dietary phosphorus on structure and composition of turbinate and long bones. Journal of Animal Science, v.30, n.5, p.742-747, 1970.

INTERNATIONAL ATOMIC ENERGY AGENCY - IAEA. Laboratory training manual on the use of nuclear techniques in animal research. Vienna: 1979. 299p. (Technical Report Series, 193).

JONGBLOED, A.W.; MROZ, Z.; KEMME, P.A. The effect of supplementary aspercylus niger phytase diets for pigs on concentration and apparent digestibility of dry sections of the alimentary tract. Journal of Animal Science, v.70, n.4, n.1, p.1159-1168, 1992.

JUNQUEIRA, O.M. Metais pesados contaminam carne. Avicultura \& Suinocultura Indutrial, n.997, p.27-29, 1993.

LOPES, J.B. Efeito dos níveis de proteínas e energia sobre características de carcaça de suínos em crescimento e terminação. In: SEMINÁRIOS DE PESQUISA AGROPECUÁRIA DO PIAUÍ, 5., 1988, Teresina. Anais... Teresina: EMBRAPA, 1988. p.209216.

LOPES, J.B. Avaliação da absorção real e das perdas endógenas de fósforo em suínos pela técnica de diluição isotópica. 1998. 87f. Tese (Doutorado em Energia Nuclear na Agricultura) - Centro de Energia Nuclear na Agricultura, Universidade de São Paulo, Piracicaba, 1998.

LOPES J.B.; VITTI, D.M.S.S.; ABDALLA, A.L. et al. Modelo do fluxo biológico do fósforo de fontes de fosfato em suínos, usando o ${ }^{32} \mathrm{P}$ como marcador. Revista Brasileira de Zootecnia, v.30, n. 1, p. $165-173,2001$.

LOPES, J.B.; VITTI, D.M.S.S.; FIGUEIRÊDO, A.V. et al. Absorção real, perdas endógenas e exigências de fósforo para suínos em final de crescimento pela técnica da diluição isotópica. Arquivo Brasileiro Medicina Veterinária Zootecnia, v.51, n.4, p.353-358, 1999a.

LOPES, J.B.; VITTI, D.M.S.S.; FIGUEIRÊDO, A.V. et al. Avaliação das perdas endógenas e das exigências de fósforo, por meio da técnica da diluição isotópica, para suínos em crescimento. Revista Brasileira de Zootecnia, v.28, n.4, p.773-778, 1999 b.

MOREIRA, J.A.; MIYADA, V.S. Fontes de fósforo para suínos. In: ARAUJO, E.T.; VIEIRA, A.C. (Eds.) Tópicos em ciências animal e pastagens. 1.ed. Piracicaba: Centro Acadêmico Luiz de Queiroz, 1998. p.387-396.

MOREIRA, J.A. Radiofósforo nos estudos de biodisponibilidade e perdas endógenas, cinética nos tecidos e modelo biomatemático, associado ao desempenho de suínos alimentados com dietas contendo fitase. 2002. 142f. Tese (Doutorado em Energia Nuclear na Agricultura) - Centro de energia Nuclear na Agricultura, Universidade de São Paulo, Piracicaba, 2002.

MOREIRA, J.A.; VITTI, D.M.S.S.; LOPES, J.B. et al. Fluxo biológico do fósforo no metabolismo de suínos alimentados com dietas contendo fitase. Revista Brasileira de Zootecnia, v.33, p.2066-2075, 2004.

NATIONAL RESEARCH COUNCIL - NRC. Nutrient requirements of poultry. 9.ed. Washington, D.C.: National Academy Press, 1994. $155 \mathrm{p}$

ROSA, I.V. Uso de rochas fosfáticas como fonte de fósforo suplementar para ruminantes. Brasília: EMBRAPA, 1983. (Parecer Técnico, 1).

ROSTAGNO, H.S.; ALBINO, L.F.T.; DONZELE, J.L. et al. Composição de alimentos e exigências nutricionais: tabelas brasileiras para aves e suínos. 2.ed. Viçosa, MG: Editora UFV, 2005. 186p.

ROSTAGNO, H.S.; SILVA, D.J.; COSTA, P.M.A. et al. Composição de alimentos e exigências nutricionais de aves e suínos: tabelas brasileiras. Viçosa, MG: Imprensa Universitária, 1987. 59p.

SARRUGE, J.R.; HAAG, H.P. Análises químicas em plantas. Piracicaba: ESALQ/USP, 1974. 56p.

STATISTICAL ANALYSIS SYSTEM - SAS. The SAS system for windows. Release 8.01. Cary: SAS Institute, 2000. (CD-ROM).

TEIXEIRA, A.O.; LOPES, D.C.; LOPES, J.B. et al. Determinação da biodisponibilidade do fósforo de diferentes fontes pela técnica de diluição isotópica, em suínos em crescimento. Revista Brasileira de Zootecnia, v.33, n.5, p.1231-1237, 2004.

VITTI, D.M.S.S.; ROQUE, A.P.; DIAS, R.S. et al. Metabolismo de cálcio em ovinos em crescimento sob suplementação com diferentes fontes de cálcio: aplicação e comparação de dois modelos matemáticos. Revista Brasileira de Zootecnia, v.35, n.6, p.2487-2495, 2006 . 